\title{
A Multi-Poller based Energy-Efficient Monitoring Scheme for Wireless Sensor Networks
}

\author{
Changlei Liu and Guohong Cao \\ Department of Computer Science \& Engineering \\ The Pennsylvania State University \\ E-mail: \{chaliu,gcao\}@cse.psu.edu
}

\begin{abstract}
For sensor networks deployed in unattended, harsh environments, the knowledge of sensor statuses such as liveness, node density and residue energy, is critical for maintaining the normal operation of the network. In this paper, we propose a poller-pollee based architecture to monitor the sensor status, focusing on two important issues: false alarm and energy efficiency. To reduce the false alarm rate, each pollee is monitored by multiple pollers. However, this approach will increase the power and bandwidth consumption. To address this issue, we propose a novel solution where the monitored sensor sends the status reports to different pollers in a round robin manner. In this way, bandwidth and power can be saved, and the false alarm rate can be reduced. We further propose a simple randomized algorithm to select the optimal number of pollers to stochastically minimize the expected total energy consumption due to monitoring. Theoretical analyses and simulations are used to demonstrate the effectiveness of the proposed techniques.
\end{abstract}

\section{INTRODUCTION}

Since sensors are often deployed in harsh and/or adversarial environments, the sensors or the communication links may fail and hence endanger the mission of the sensor network [1]. Therefore, sensor status (such as liveness, density estimation, residue energy, etc.) has to be closely monitored and made known to the sink, which can promptly react to sensor status changes.

In distributed systems, the only way to learn the status of a node is through receiving message from the node. For example, in IP networks, the poller-pollee structure [7] has been widely used for network management, where some specialized nodes are called pollers and the other nodes are called pollees. Each poller monitors its pollees by actively sending a "ping" message and then waiting for the reply (as in Fig. 1a) or by passively waiting for the pollees to send messages periodically (as in Fig. 1b). In this paper, we revisit the poller-pollee structure and apply it to sensor network. We let sensors self-organize themselves into two tiers, with pollees in the lower tier and pollers in the upper tier. The pollees send status reports to the pollers, which in turn generate aggregated status reports and forward them towards the sink. Different from existing work on poller-pollee structure, we consider two important issues: fault-tolerance and energy efficiency, when building the sensor network monitoring system.

Our contribution is twofold. First, we propose a multi-poller based monitoring scheme, where each pollee is associated with multiple pollers. Although using multiple pollers can be

This work was supported in part by the National Science Foundation (CNS0519460 and CNS-0519390). fault tolerant, sending status reports to multiple pollers at the same time can significantly increase the power and bandwidth consumption. We thus propose a novel solution where the monitored sensor sends the status reports to different pollers in a round robin manner. Status reports from different pollers can thus be combined to reduce false alarm. It is shown that the round robin based multi-poller solution can significantly reduce the false alarm rate compared to the single poller scheme, with similar bandwidth consumption. Second, because energy efficiency is a major concern in sensor networks, we formulate the problem of minimizing the monitoring energy consumption as a minimum energy many-to-many poller-pollee assignment problem. A simple randomized algorithm is proposed to select the optimal number of pollers to stochastically minimize the expected total energy consumption due to monitoring.

The rest of the paper is organized as follows. Section II presents the two-tier monitoring architecture and formulates the problem. The round robin based multi-poller scheme is presented and analyzed in Section III. In Section IV, we propose the randomized algorithm and derive the optimal number of pollers to minimize the expected energy consumption. Section V gives the evaluation results, and Section VI concludes the paper.

\section{ARChitecture And Problem Formulation}

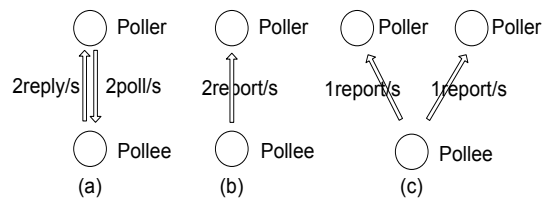

Fig. 1. Basic and extended poller-pollee structure

The poller-pollee structure has been widely deployed in IP networks for monitoring purpose. However, traditional pollerpollee structure like Fig. 1(a) and Fig. 1(b) is not resilient to node or communication failure because each pollee is associated with only one poller. To tolerate these failures, we extend the poller-pollee structure by associating each pollee with multiple pollers, as shown in Fig. 1(c).

Our monitoring architectures is built upon the multi-poller structure in Fig. 1(c). Given the scale of sensor networks, a centralized polling scheme is not scalable. Therefore, we propose a two-tier distributed polling structure where pollees send status reports to the pollers, which in turn generate aggregated status reports and forward them to the sink. We assume all sensors are capable of being either pollers or 


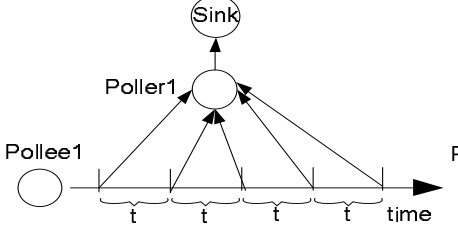

(a) the single-poller scheme

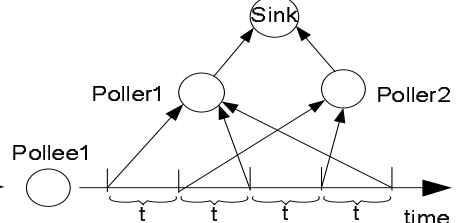

(b) the multi-poller scheme
Fig. 2. Asynchronous operation at poller and pollee, with the arrow denoting the status reporting

pollees. We further assume the aggregation is done only at poller based on the collective data within a polling domain, and transmission of one packet over one hop consumes one unit of energy.

Based on above architecture and assumptions, the minimum energy poller selection and pollee assignment problem minPL can be stated as follows. Given a network graph, determine (1) which nodes to be the pollers and which nodes to be the pollees, (2) a many-to-many mapping among pollers and pollees, such that the distance between poller and pollee is bounded by $k$ hops, and the total energy due to monitoring is minimized.

The problem minPL is proven to be NP-hard via a reduction from the minimum $k$-hop dominating set problem [4]. A $k$ hop dominating set of a graph $\mathrm{G}$ is a set of vertices such that for every vertex $u$ of $\mathrm{G}$, there is a vertex $v$ in the dominating set and the distance between $u$ and $v$ is at most $k$. To see the equivalence of the two problems, we consider a special case of minPL, where the aggregation ratio $s=1$ and each pollee is associated with $\omega=1$ poller. Further suppose the sink is far away enough from the region to be monitored, so the energy spent in communicating the data from the poller to the sink dominates the total every consumption. Then, minimizing the total energy consumption becomes minimizing the number of pollers, which is exactly the cardinality of $k$-hop dominating set. Since the problem of minimum $k$-hop dominating set is NP-complete and it reduces to minPL, minPL is NP-hard.

\section{THE ROUND ROBIN BASED MULTI-POLLER SCHEME AND ITS PERFORMANCE ANALYSIS}

\section{A. The Round Robin Based Multi-Poller Scheme}

Recall that the multi-poller structure is proposed to deal with sensor and communication link failures. To reduce the communication overhead, we propose a round robin based multi-poller scheme, where the monitored sensor sends the status report to different pollers in a round robin manner. Status reports from different pollers can thus be combined to reduce false alarm rate. For example, even the report through one poller is lost due to transient communication link failure, the sink may still receive the pollee's report from another poller in the next time interval.

Let $t$ denote the polling time interval at the pollee, and let $\omega$ denote the number of pollers for each pollee. Suppose the sink needs time $T_{d}$, referred to as the detection time, to detect a pollee failure. The single-poller and multi-poller scheme can be stated as follows:

- Single-poller scheme: each pollee sends a report to the same poller every $t$; each poller collects reports from its pollees every $t$ and forwards the compressed report to the sink every $t$; the sink evaluates the failure condition every $T_{d}$. It is required that $t \leq T_{d}$.

- Multi-poller scheme: each pollee schedules a report to different pollers every $t$; each poller collects reports from each of its pollees every $\omega t$ and forwards the compressed report to the sink every $\omega t$; the sink evaluates the failure condition every $T_{d}$. It is required that $\omega t \leq T_{d}$.

Fig. 2 illustrates the single poller scheme and multi-poller scheme using an example. In Fig. 2(a), pollee 1 sends reports to poller 1 every $t$; in Fig. 2(b), pollee 1 sends reports to poller 1 or poller 2 every $2 t$ in a round robin manner. The sink receives two reports every $2 t$ if it chooses detection time $T_{d}=$ $2 t$. With the single poller scheme, if some communication link between pollee 1 and the sink has some problem, the sink will have a false alarm on the status of pollee 1 . However, there will not be any false alarm in the multi-poller scheme. In general, with the same detection time delay and the same amount of traffic, the multi-poller scheme will have a much smaller false alarm rate on the status of the pollee.

\section{B. The False Alarm Rate}

In wireless medium, the characteristics of node failure and link failure are quite different. While the node failure may be permanent, the wireless link failure happens from time to time, and once occurs it will persist for a period of time. During the transmission of messages, if an intermediate node is detected to fail, its downstream nodes can simply bypass it by choosing an alternative next hop. But the same technique cannot be applied to the case of link failure, which is transient in nature. Therefore we study the link failure and model it as a continuous-time Markov chain, as adopted by some existing work [10]. The following notations are used in the analysis:

$f_{l}$ : link failure rate

$h$ : number of hops from pollee to poller

$F_{1}\left(h, T_{d}\right), F_{w}\left(h, T_{d}\right)$ : the false alarm rate for the single poller scheme, and the multi-poller scheme, where the subscript $w$ represents the number of pollers for each pollee.

Each link is modeled as a two state markov chain (Fig. 3a). The transition rate from state 0 ("on") to state 1 ("off") and from state 1 to state 0 is $\lambda$ and $\mu$ respectively. If the path between the poller and pollee is multihop, it can be modeled by the higher dimensional markov chain. For example in Fig. 3(b), the two-hop path (i.e., $h=2$ ) is represented by four states with "11" denoting the state at which both links fail. Further assume each pollee has $\omega$ pollers $(\omega \geq 1)$, and a packet is sent to each poller every $\omega t$ period. We are interested in the false alarm rate $F_{w}\left(h, T_{d}\right)$, given $h$ and the detection period $T_{d}$.

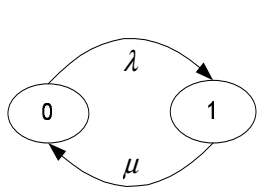

(a) $\mathrm{h}=1$

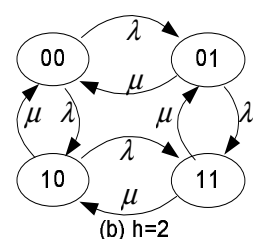

(b) $\mathrm{h}=2$
Fig. 3. Continuous-time markov chain link model 
- The continuous-time markov chain of a link (Fig. 3a): To solve the markov chain in Fig. 3(a), its limiting probability $\pi_{0}, \pi_{1}$, i.e., the long-run proportion of time that the process is in state 0 and state 1 , can be calculated by $\pi_{0}=\frac{\mu}{\lambda+\mu}, \pi_{1}=\frac{\lambda}{\lambda+\mu}$. The transition probability $p_{i j}(t)$, the probability that the state is in $j$ at time $t$ provided that the current time is $i$, is given by:

$$
\begin{aligned}
& p_{00}(t)=\frac{\lambda}{\lambda+\mu} e^{-(\lambda+\mu) t}+\frac{\mu}{\lambda+\mu} \\
& p_{10}(t)=\frac{\mu}{\lambda+\mu}-\frac{\mu}{\lambda+\mu} e^{-(\lambda+\mu) t}
\end{aligned}
$$

- The multihop path with single poller (Fig. 3b): Without loss of generality, we assume $T_{d} / t$ is an integer and discretize the time using interval $t$. Then $F_{1}\left(h, T_{d}\right)$ can be calculated as follows.

1) When $T_{d}=t$,

$$
F_{1}(h, t)=1-\pi_{0}(t)^{h}, \text { for } \forall h
$$

2) When $T_{d}=2 t, h=2$,

$$
\begin{aligned}
F_{1}(2,2 t) & =\pi_{1} \pi_{0}\left(1-p_{10}(t) p_{00}(t)\right) \\
& +\pi_{0} \pi_{1}\left(1-p_{00}(t) p_{10}(t)\right) \\
& +\pi_{1} \pi_{1}\left(1-p_{10}(t) p_{10}(t)\right)
\end{aligned}
$$

Above calculation numerates the three cases of false alarm in which the two-hop path fails in both $t$ intervals.

3) When $T_{d}=2 t, h=3$,

$$
\begin{array}{r}
F_{1}(3,2 t)=\sum_{i=0}^{1} \sum_{j=0}^{1} \sum_{k=0}^{1} \pi_{i} \pi_{j} \pi_{k}\left(1-p_{i 0}(t) p_{j 0}(t)\right. \\
\left.p_{k 0}(t)\right)-\pi_{0}^{h}\left(1-p_{10}(t) p_{10}(t) p_{10}(t)\right)
\end{array}
$$

4) Eqn. 5 can be generalized to calculate $F_{1}(h, 2 t)$

5) In general, $F_{1}\left(h, T_{d}\right)$ can be calculated by

$$
\begin{aligned}
& F_{1}(h, t) \quad\left(P\left[s\left(t+t_{0}\right)=1 \mid s\left(t_{0}\right)=1\right]\right)^{\frac{T_{d}}{t}-1} \\
= & F_{1}(h, t) \quad\left[\frac{\left(P\left[s\left(t+t_{0}\right)=1, s\left(t_{0}\right)=1\right]\right)}{P\left[s\left(t_{0}\right)=1\right]}\right]^{\frac{T_{d}}{t}-1} \\
= & F_{1}(h, t)\left[\frac{F_{1}(h, 2 t)}{F_{1}(h, t)}\right]^{\frac{T_{d}}{t}-1}
\end{aligned}
$$

where $s\left(t_{0}\right)$ denotes the path state at $t_{0}, P[s(t+$ $\left.\left.t_{0}\right)=1 \mid s\left(t_{0}\right)=1\right]$ is the transition probability, and $F_{1}(h, t), F_{1}(h, 2 t)$ are given by 1$\left.), 4\right)$. Above follows because the markov chain is homogeneous, i.e., its transition probability does not depend on the starting time.

- Multihop path with multi-poller: Unlike the single poller scheme, the failure of the multihop paths towards different pollers are independent. Therefore $F_{\omega}\left(h, T_{d}\right)$ is given by:

$$
F_{\omega}\left(h, T_{d}\right)=\left(1-\pi_{0}(t)^{h}\right)^{T_{d} / t}
$$

Fig. 4 shows the false alarm rate as a function of the number of hops from poller to pollee when $f_{l}=0.1$. As can be seen, the false alarm rate increases as $h$ increases, since longer path is more vulnerable to failure. As $T_{d}$ increases, the false alarm rate decreases. For example, even for the single poller scheme, when $h=1, F_{1}(h, t)=0.1$, but $F_{1}(h, 2 t)=0.025$; that is, doubling the detection time can reduce the false alarm rate by as much as $75 \%$. We can also see that the multi-poller scheme consistently outperforms the single poller scheme in terms of false alarm rate. For example, with $h=1, F_{1}(h, 2 t)=0.025$, but $F_{2}(h, 2 t)=0.01$, which represents a $60 \%$ false alarm reduction. This is because the status report of the multi-poller scheme is transmitted to the sink through different paths, and hence has better tolerance for the link failure at any path. Note that, due to the use of round robin scheme, the multi-poller scheme still keep similar bandwidth consumption as the single poller scheme.

\section{An EnERGy EFFICIENT Randomized Algorithm}

\section{A. The Randomized Algorithm}

A simple randomized algorithm is proposed to select the statistically optimal number of pollers to minimize the average energy consumption. Each node first elects itself as a poller with probability $p$. The elected pollers then announce their poller status within $k$ hops. Sensor nodes that did not elect themselves as pollers will be pollees, and each pollee selects $\omega$ monitoring pollers based on the received announcements. For easy presentation, we set $\omega=2$ although the proposed solution can be generalized to $\omega \geq 2$. The value of $k$ is carefully chosen to ensure that most pollees can locate their primary and second pollers with a high probability. In case that some unlabeled node does not receive poller declaration within a designated period of time, it waits for a random amount of time and elects itself as a poller. The random timer is used to void cases that neighboring nodes elect themselves simultaneously.

Similar to AODV [8], the poller announcement contains poller identifier, sequence number and the current hop count. These information can make sure that a selected path from the pollee to the poller is loop free. Based on the received poller announcement, a pollee always picks the closest poller as the primary poller.

After establishing the primary poller-pollee relationship, a secondary poller should be chosen for each pollee. To be fault tolerant, the primary path (from the pollee to the primary poller) and the secondary path (from the pollee to the secondary poller) is better to be node disjoint. Disjoint path selection is straightforward if the path information can be added into the poller announcement message. Otherwise, we can utilize some existing schemes such as [11], whose goal is to find multiple disjoint paths to different destination nodes, as in our problem.

\section{B. Finding the Optimal Number of Pollers}

In this subsection, we calculate the optimal poller election probability $p^{*}$ for the randomized algorithm. We assume $n$ sensors are uniformly deployed in a $2 a \times 2 a$ square area. Based on the spatial point theory [6], when $n$ is large and the deployment follows uniform distribution, the spatial location of the sensors can be approximately governed by a homogeneous two-dimension Poisson process. The Poisson assumption for large scale sensor network has been adopted by many existing 


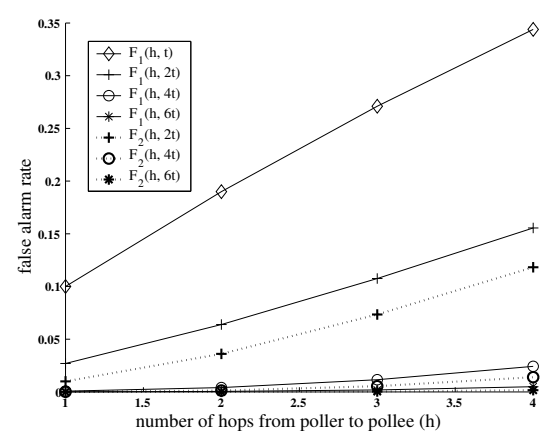

Fig. 4. False alarm rate $\left(f_{l}=0.1\right)$

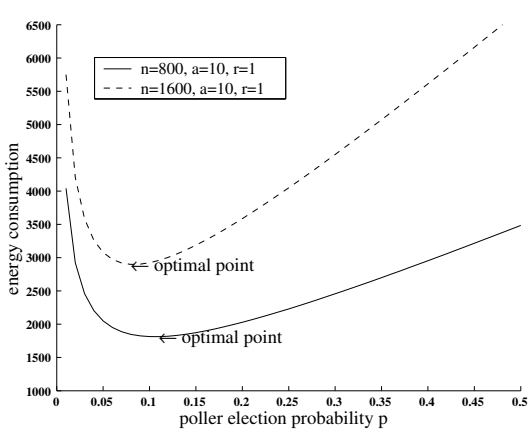

Fig. 5. Energy VS. $p(\omega=2)$

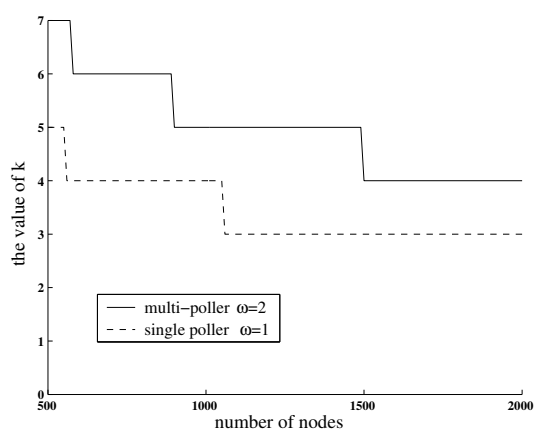

Fig. 6. The $k$ value VS. $n$ works such as [3], [9], [5]. The following notations are used for the analysis.

$\Lambda$ : parameter of the homogeneous Poisson process. The number of sensors within area $A$ is a Poisson random variable with mean $\Lambda A$.

$p$ : the poller election probability.

$p^{*}$ : optimal value of $p$ to minimize the energy consumption.

$r$ : fixed transmission radius. In large sensor networks, a distance of $d$ can be approximated by $\lceil d / r\rceil$ hops.

$s$ : aggregation ratio. $s$ status reports can be aggregated into a single packet.

Our objective is as follows: given a network of $n$ nodes, together with the parameters $a, \Lambda, r, s$, we want to find $p^{*}$ such that the average energy consumption per polling interval, denoted by $E[C]$, can be minimized if each pollee is monitored by $\omega$ pollers. $E[C]$ below is derived using some geometrical results on Voronoi Diagram [2], [9].

$$
E[C]=\frac{n(1-p)}{2 r \Lambda^{1 / 2} p^{1 / 2}}+\frac{(2 \sqrt{2}-1)-(\sqrt{2}-1) p}{4 r \Lambda^{1 / 2} p^{1 / 2}}+\frac{0.765 n p a}{r}
$$

To minimize $E[C], \frac{d E[C]}{d p^{*}}=0 \Rightarrow a\left(\sqrt{p^{*}}\right)^{3}+b\left(\sqrt{p^{*}}\right)^{2}+d=$ 0 , where $a=3.06 a \Lambda^{1 / 2}, b=-\sqrt{2} / 2, d=-\sqrt{2}$

The only real root of the above equation is

$$
\begin{gathered}
\sqrt{p^{*}}=\frac{2^{1 / 3} b^{2}}{3 a\left[-2 b^{3}-27 a^{2} d+\sqrt{-4 b^{6}+\left(2 b^{3}+27 a^{2} d\right)^{2}}\right]^{1 / 3}} \\
+\frac{\left[-2 b^{3}-27 a^{2} d+\sqrt{-4 b^{6}+\left(2 b^{3}+27 a^{2} d\right)^{2}}\right]^{1 / 3}}{3 \times 2^{1 / 3} a}-\frac{b}{3 a}
\end{gathered}
$$

Following [9], [3], we derived the formula for $k$, such that within $k$-hop the pollee can find the primary and secondary poller with a probability no less than $(1-\alpha)$ :

$$
k=\left\lceil\frac{1}{r} \sqrt{\frac{-1.834 \ln \frac{\alpha}{7}}{p^{*} \Lambda}}\right\rceil
$$

Figs. 5, 6 show some numerical results on the relationship between $p, k$, the number of sensors, and the total energy consumption. As seen in Fig. 5, the energy consumption is very high when $p$ is very small or very large. When $p$ is very small, there are only a small number of pollers. Many pollees have to send their status reports to a far away poller, and some of them may go through multiple hops, consuming lots of energy. When $p$ is too large, there will be many pollers, but each poller only has a very limited number of pollees. Thus, the power consumption of sending the status report from the poller to the sink is very high. From the figure, we can see that there exists an optimal $p^{*}$, which can be used to minimize the energy consumption.

Fig. 6 further studies how the value of $k$ changes with $n, \omega$. Recall that $k$ is the number of hops that the poller announcement will be broadcast such that every pollee can find a poller with a certain probability. In general, $k$ decreases as $n$ increases, and $k$ increases as $\omega$ increases. Intuitively, as $n$ increases, more pollers are elected, thus a smaller $k$ is sufficient to cover all the pollees with high probability; as $w$ increases, i.e., more pollers are assigned to each pollee, $k$ needs to increase to cover a wider range.

\section{Performance Evaluations}

In this section, we verify the analytical results and compare the performance of the single poller and multi-poller schemes through simulations. In the simulation, $n$ sensors are randomly deployed in a $20 \times 20$ square area. For the multi-poller scheme, each pollee sends status reports to the associated $\omega$ pollers in a round robin manner. The transmission of one packet over one hop is assumed to consume one unit of energy. The link failure is modeled as a continuous-time markov chain with an average failure rate of $f_{l}$. We set the aggregation ratio $s=\left\lfloor\frac{29}{4}\right\rfloor=7$. This is because in Mica2 sensor motes, each packet has a 29byte payload, and we assume the status information such as the residue energy can be represented by 4 bytes.

\section{A. The Effects of parameters $p$ and $k$}

Fig. 7 shows the effects of $p$ on the total energy consumption. On each curve, the real optimal $p^{*}$ corresponds to the point of minimum energy, while the depicted optimal point corresponds to the numerical result derived in Subsection IVB. It can be seen that the energy consumption in the simulation is larger than that in the analysis. The mismatch may be due to the assumption that is used in the analysis to approximate the routing hops by the distance divided by the transmission range. However, the numerical and simulation results have the same trend, and a good match on the optimal point $p^{*}$ can be observed. In the following experiments, we set $p=p^{*}$.

$k$ is the number of hops that the poller announcement is broadcast. It should be carefully chosen to balance the tradeoff between the announcement coverage and message complexity. In Fig. 8, the relationship between the value of $k$ and the energy consumption is studied. It is shown that the average 


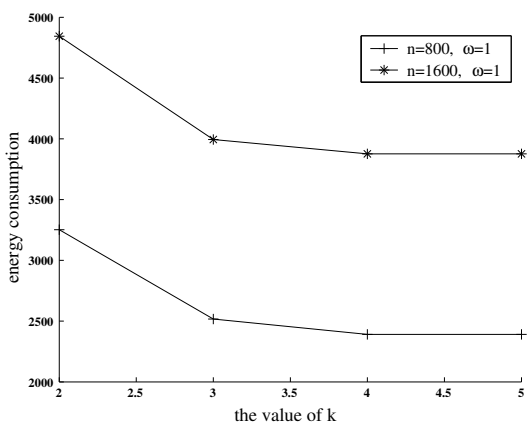

Fig. 8. Effect of $k$ on energy consumption

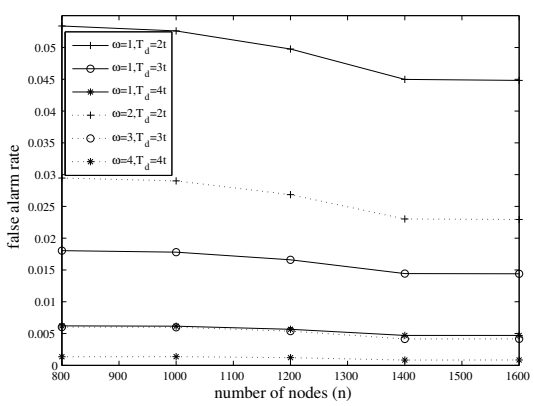

Fig. 9. Comparison of single and multi-poller scheme (with link failure $f_{l}=0.1$ )

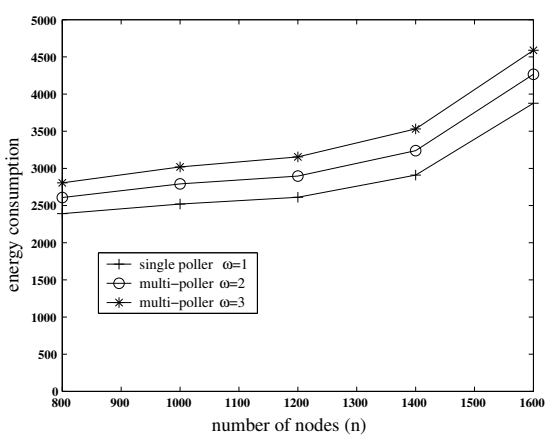

Fig. 10. Energy comparison of single and multipoller scheme (without failure)

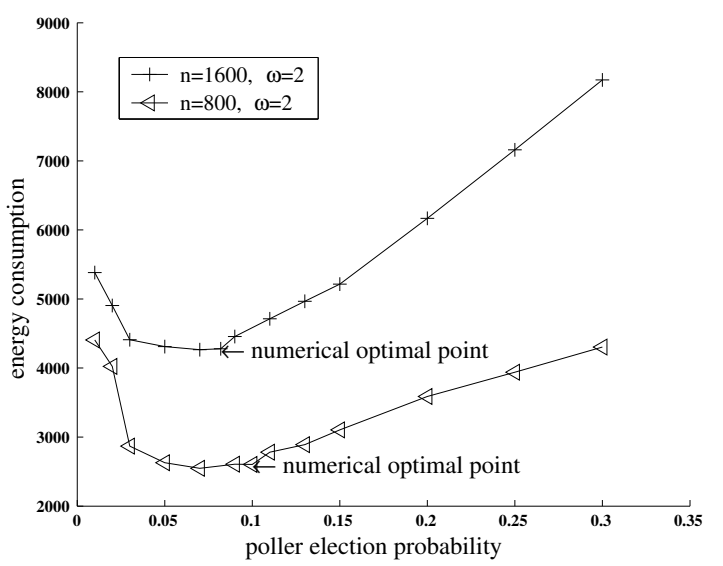

Fig. 7. Energy VS. $p(\omega=2)$

energy consumption is the highest when $k=2$ but gradually decreases as $k$ increases from 3 to 5 . This is because when $k$ is too small, a good portion of pollees cannot receive poller announcement, and thus become poller themselves. At this time, the number of pollers is no longer optimal, and hence the performance degrades.

\section{B. Comparison of Single Poller and Multi-Poller Schemes}

Fig. 9 compares the single poller scheme with the multipoller scheme in presence of link failure. For fair comparison, the same detection period $T_{d}$ is used. For example, $T_{d}=4 t$ implies that four reports are sent out within the detection period $T_{d}$. From Fig. 9, we have several observations. First, consistent with the numerical result, the false alarm rate dramatically drops as the detection period $T_{d}$ increases. This verifies the tradeoff between the detection delay and the false alarm rate. Second, the reduction of the false alarm rate in the multi-poller scheme is over 50\% compared to that of the single-poller scheme, irrespective of the number of nodes. As discussed below, the substantial performance improvement is at the cost of a marginal increase of the bandwidth consumption (shown in Fig. 10).

Fig. 10 studies the energy cost associated with the multipoller scheme. It costs $7 \%$ more energy when the number of pollers for each pollee increases from 1 to 2 or from 2 to 3 . Based on our randomized algorithm, the primary path is always the shortest path, but the secondary path may be a little bit longer. As a result, packets may go through more hops to reach the secondary poller compared to that of the primary poller, resulting in more energy consumption. However, compared with the improvement on false alarm rate as shown in Fig. 9, the energy increase is pretty small.

\section{Conclusions}

In this paper, we proposed solutions to address challenges in monitoring wireless sensor networks; i.e., false alarm, energy efficiency, and distributed monitoring. In the proposed monitoring system, sensors self-organize themselves into two tiers, with pollees in the lower tier and pollers in the upper tier. The pollees send status reports to the pollers, which in turn generate aggregated status reports and forward them towards the sink. To reduce the false alarm rate, we have proposed a multi-poller based structure where pollees send reports to the pollers in a round robin manner. To be energy efficient, we have presented a distributed randomized algorithm which can select the optimal number of pollers to minimize the total expected monitoring traffic. Theoretical analyses and simulations demonstrated the effectiveness of the proposed techniques.

\section{REFERENCES}

[1] I. Akyildiz, W. Su, Y. Sankarasubramaniam, and E.Cayirci, "Wireless Sensor Networks: A Survey," Computer Networks, 2002.

[2] Franz Aurenhammer, "Voronoi diagrams: a survey of a fundamental geometric data structure," ACM Computing Surveys (CSUR), 1991.

[3] S. Bandyopadhyay and E. Coyle, "An energy-efficient hierarchical clustering algorithm for wireless sensor networks," in INFOCOM, 2003.

[4] F. Dai and J. Wu, "On constructing k-connected k-dominating set in wireless networks," JPDC, July 2006.

[5] C. Gui and P. Mohapatra, "Power conservation and quality of surveillance in target tracking sensor networks," in MOBICOM, 2004.

[6] P. Hall, Introduction to the Theory of Coverage Processes, New York: Wiley, 1998.

[7] Li (Erran) Li, Marina Thottan, Bin Yao, and Sanjoy Paul, "Distributed network monitoring with bounded link utilization in ip networks," in INFOCOM, San Francisco, April 2003.

[8] C. Perkins and E. Royer, "Ad-Hoc On Demand Distance Vector Routing," in IEEE WMCSA, February 1999.

[9] S.G.Foss and S.A. Zuyev, "On a voronoi aggregative process related to a bivariate poisson process," Advances in Applied Probability, 1996.

[10] S. Sivaprakasam and K.S. Shanmugan, "An equivalent markov model for burst errors in digital channels," IEEE Trans. Commun., 1995.

[11] Preetha Thulasiraman, Srinivasan Ramasubramanian, and Marwan Krunz, "Disjoint multipath routing to two distinct drains in a multidrain sensor network," in INFOCOM, 2007, pp. 643-651. 